\title{
Isolation of Plastoquinone from Spinach by HPLC
}

\section{Marco Malferrari* and Francesco Francia}

Laboratorio di Biochimica e Biofisica, Dipartimento di Farmacia e Biotecnologie, FaBiT, Università di Bologna, 40126 Bologna, Italy

\begin{abstract}
We report a method for the purification of plastoquinone-9 (PQ), a prenylquinone cofactor involved in the photosynthetic electron transport chain. The described procedures relies on spinach-chloroplast isolation followed by $P Q$ extraction and chromatographic fractionation. Extraction of $P Q$ was achieved using partition of chloroplast suspension with methanol:petroleum ether. This procedure removed large amounts of green pigments from the extract and thus facilitates the subsequent chromatographic isolation of $P Q$. To obtain pure $P Q$, the developed extraction was combined with a two-step chromatographic approach using orthogonal stationary phases, i.e. alumina and octadecylsilane (C18). A small scale protocol for analytical reverse-phase high performance liquid chromatography (RP-HPLC), which may be implemented in most laboratories equipped with conventional systems, is described. The reported methodology represents a valuable tool for the fast production of small amounts of $P Q$, for which there are no commercial standards available.
\end{abstract}

Keywords: Spinacia oleracea, plastoquinone-9, HPLC.

\section{Introduction}

In plants, algae and cyanobacteria the light-driven redox reactions responsible for the primary energy transduction by photosynthesis involve three major membrane protein complexes named photosystem II (PSII), cytochrome $b_{6} f$, and photosystem I (PSI). Active centers of these complexes communicate via two redox carriers: membranesoluble plastoquinone-9 (PQ) connects the reducing activity of PSII to the oxidizing activity of the $b_{6} f$ complex, while the soluble protein plastocyanin, is reduced by the $b_{\sigma} f$ activity and oxidized by PSI $[1,2]$.

Besides its main role as proton and electron carrier, PQ is an active player in the short and long term light adaptation responses of photosynthetic cells [3-6]. Recently the involvement of PQ as singlet oxygen scavenger has been reported in high light stressed Chlamydomonas reinhardtii cultures [7].

In spite of the reborn interest for the different PQ functions, which are at present intensively investigated, PQ reference standards are no longer commercially available and the purification methods reported in the literature are often fragmentary and dated, being based on protocols published in the 60-80's [8-11]. In the present paper we describe a procedure in which PQ is directly extracted from water suspensions of chloroplasts by partition with methanol:petroleum ether. The enriched extract is then chromatographed on two orthogonal phases for maximum selectivity using alumina and reverse phase columns. The described protocol, which allows straightforward isolation of pure PQ from spinach chloroplasts, is applied on a small scale requiring HPLC instrumentation normally present in a laboratory.

\section{Materials and Methods}

\section{Chloroplast preparation and solvents}

Chloroplasts were prepared from spinach leaves (Spinacia oleracea L.) following standard protocols described in Barr and Crane [8]. The total chlorophyll $(\mathrm{Chl})$ content was determined spectrophotometrically upon extraction of a few $\mu \mathrm{L}$ of chloroplast suspension with acetone, accordingly to Arnon [12]. Acetone, methanol, petroleum ether (bp $40-60^{\circ} \mathrm{C}$ ), diethyl-ether, ethanol and acetonitrile of analytical or HPLC grade, used as solvent for PQ extraction and isolation, were from Sigma-Aldrich.

\section{Extraction and fractionation of plastoquinone}

As an alternative to the Barr and Crane method [8], we tested for PQ extraction from chloroplasts a protocol set up by Venturoli et al. [13] for the extraction of ubiquinone- 10 from membranes of photosynthetic bacteria. To this aim, $100 \mathrm{~mL}$ of sugar free chloroplasts were diluted in water to a final Chl concentration $(\mathrm{a}+\mathrm{b})$ of $1 \mathrm{mg} / \mathrm{mL}$. A 20 time volume excess of a methanol:petroleum ether 3:2 mixture was directly added to the liquid chloroplast sample and vigorously shaked for $1 \mathrm{~min}$. After phase separation, the petroleum ether was collected, and the extraction repeated twice by re-adding fresh petroleum ether to the methanolwater lower phase. The petroleum ether fractions were combined, dried by rotary evaporation and resuspended in $10 \mathrm{~mL}$ of petroleum ether.

The extract obtained was fractionated by open column chromatography on acid washed alumina (SIGMA-Aldrich) following the scheme of Barr and Crane [8]. $100 \mathrm{~g}$ of alumina were deactivated by adding $6 \mathrm{ml}$ of double distilled (d.d.) water, resuspended in petroleum ether and placed into a glass column with diameter and length of 2.5 $\mathrm{cm}$ and $40 \mathrm{~cm}$, respectively. The petroleum ether extract, containing $\mathrm{PQ}$, was loaded into the column and 7 fractions (1-7) were obtained by eluting in sequence with $100 \mathrm{~mL}$ of $0,0.2,2,4,8,16$ and $20 \%$ of diethylether in petroleum ether $(\mathrm{v} / \mathrm{v})$.

\section{HPLC plastoquinone isolation}

Analytical HPLC analysis was performed at $40^{\circ} \mathrm{C}$ by using a Jasco Pu-1580 pump, a C-18 reverse phase column (Waters Spherisorb $5 \mu \mathrm{m}$ ODS2, $4.6 \times 250 \mathrm{~mm}$ ), and a Jasco UV 970 detector operating at 255 or $290 \mathrm{~nm}$. The mobile phase (flow rate $1.5 \mathrm{~mL} / \mathrm{min}$ ) was a mixture of acetonitrile:ethanol 3:1, as described in Yoshida et al. [14].

*Corresponding author: Marco Malferrari, Laboratorio di Biochimica e Biofisica, Dipartimento di Farmacia e Biotecnologie, FaBiT, Università di Bologna, 40126 Bologna, Italy, Tel: +39 0512091293; Fax: +39 051242576 ; E-mail: marco.malferrari2@unibo.it

Received September 19, 2014; Accepted October 24, 2014; Published October 31,2014

Citation: Malferrari M, Francia F (2014) Isolation of Plastoquinone from Spinach by HPLC. J Chromatogr Sep Tech 5: 242. doi:10.4172/2157-7064.1000242

Copyright: (c) 2014 Malferrari M, et al. This is an open-access article distributed under the terms of the Creative Commons Attribution License, which permits unrestricted use, distribution, and reproduction in any medium, provided the original author and source are credited. 
UV-visible absorption spectra of isolated PQ were recorded using a Jasco V-550 UV/VIS spectrophotometer.

\section{Results and Discussion}

The method of PQ purification described in the present work is discussed in the following by considering separately: (i) the extraction with organic solvents; (ii) the fractionation by aluminium oxide; (iii) the isolation by HPLC and recovery of pure components.

\section{Extraction with organic solvent}

From $500 \mathrm{~g}$ of spinach leaves, $95 \mathrm{~mL}$ of chloroplasts containing chlorophyll $(\mathrm{a}+\mathrm{b})$ at a concentration of $1.34 \mathrm{mg} / \mathrm{mL}$ were obtained. After washing chloroplasts in water to get a sugar free chloroplast suspension, at variance to established protocols [8], extraction of PQ was achieved by partition between a methanol:water phase and a petroleum ether phase (see Materials and Methods for details). This procedure, extensively used for ubiquinone extraction form chromatophores of photosynthetic bacteria [13], was found to be effective also in the case of PQ. Although a small portion of PQ remained in the methanol:water phase, resulting in a slightly lower extraction yield as compared to the established method [8], this extraction procedure provided significant advantages. First time-consuming chloroplast lyophilization could be avoided, which speeded up the procedure and prevented possible unwanted reactions during lyophilization. Second, since large amounts of green pigments remained trapped in the methanol-water phase, the subsequent purification steps were considerably facilitated. In order to further separate PQ from chlorophyll and other pigments, the obtained extracts were then treated on an aluminium oxide column as described below.

\section{Fractionation by aluminium oxide}

The use of an acidic alumina column, besides removing to a large extent green pigments and carotenoids, has the advantage that plastoquinone oxidation takes place during this procedure. Indeed it has been shown $[15,16]$ that a substantial fraction $(30-40 \%)$ of the extracted plastoquinone is in the doubly reduced and protonated form, i.e. plastoquinol $\mathrm{PQH}_{2}$. The conversion to the more stable oxidized form facilitates the separation and the collection of the purified PQ.

The residue from the methanol:petroleum ether extraction was loaded on the top of a $100 \mathrm{~g}$ aluminium oxide column and eluted with fractions of $100 \mathrm{~mL}$ petroleum ether with increasing v/v percentages of diethyl ether (see Extraction and fractionation of plastoquinone). At variance to what reported in established protocols [8], PQ eluted in fraction 5. This difference in the elution profile was likely due to the much lower concentration of green pigments in the methanol:petroleum ether extract. To maximize the final yield of PQ, an HPLC confirmatory analysis of the fractions (from 1 to 7 ) should be, however, always done to ascertain the presence of $\mathrm{PQ}$ in the respective fractions.

\section{Isolation and recovery of plastoquinone}

The dried residue of the eluted fraction 5 from the aluminium oxide column was dissolved in ethanol and subjected to HPLC analysis; the obtained chromatograms are shown in Figure 1. Oxidized PQ is known to present an absorption maximum at $255 \mathrm{~nm}\left(\varepsilon_{255}=17.94\right.$ $\left.\mathrm{mM}^{-1} \mathrm{~cm}^{-1}\right)$ that shifts to $290 \mathrm{~nm}$ upon reduction $\left(\varepsilon_{290}=3.39 \mathrm{mM}^{-1} \mathrm{~cm}^{-1}\right)$ [17]; this implies that the detection of the oxidized or the reduced PQ peaks are maximized at $255 \mathrm{~nm}$ and $290 \mathrm{~nm}$, respectively. As shown in the upper panel of Figure 1, a large peak with a retention time of 19.2 minutes is present in the chromatogram at $255 \mathrm{~nm}$ (black trace), characteristic of the oxidized PQ [14]. Essentially no peak appeared in

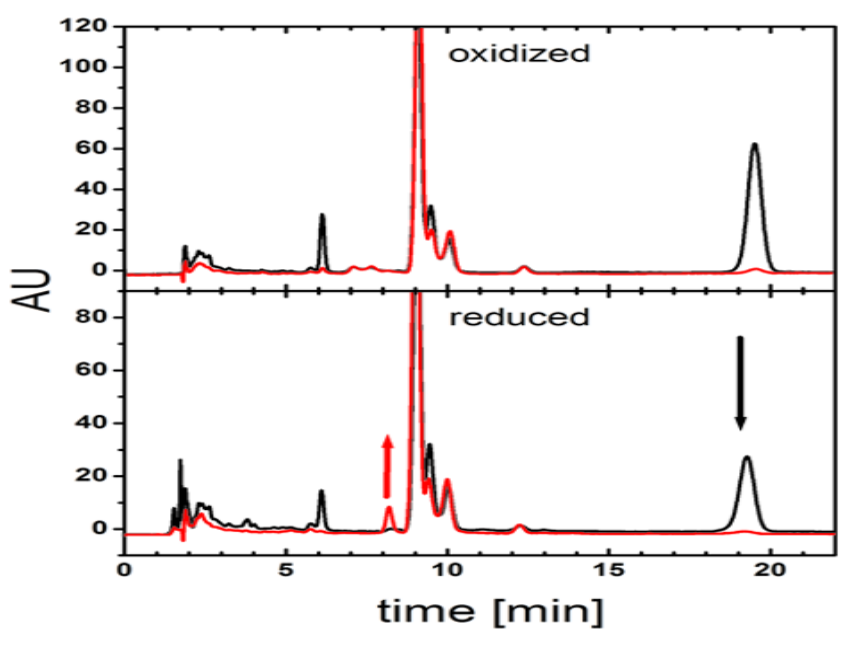

Figure 1: HPLC elution profiles after fractionation with aluminium oxide. Upper panel: chromatogram of the alumina column fraction 5 from the methanol : petroleum ether extract of water suspended chloroplasts. Red and black chromatograms refer to elution profiles detected at 290 or $255 \mathrm{~nm}$, respectively. In the lower panel, black downward arrow indicates the elution of the $P Q$ oxidized form (retention time of $19.2 \mathrm{~min}$ ); the peak of the oxidized form at 255 $\mathrm{nm}$ is decreased upon reduction. Red upward arrow indicates the elution of the $P Q$ reduced form (retention time of $8.1 \mathrm{~min}$ ) whose peak at $290 \mathrm{~nm}$ becomes visible upon reduction.

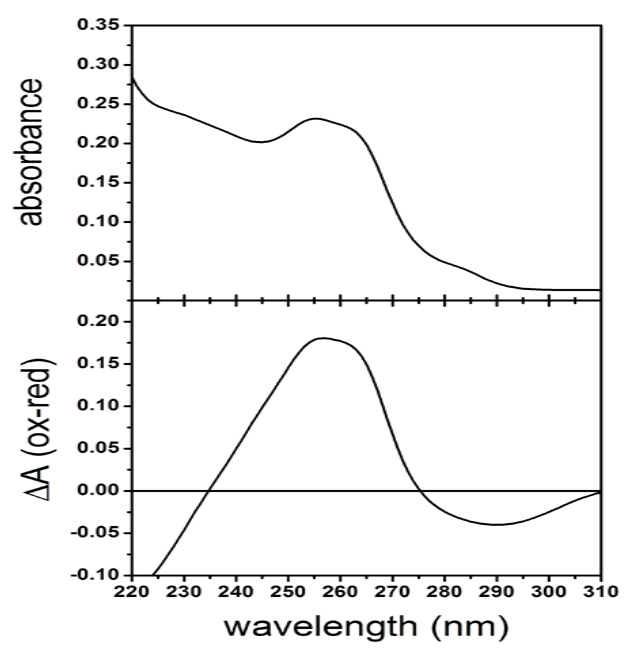

Figure 2: UV spectra of HPLC purified PQ.

Upper panel: spectrum of PQ collected from the HPLC. Lower panel: oxidizedreduced difference spectrum. The oxidized PQ eluting at 19.2 min (see Figure 1) has been collected from analytical HPLC runs, dried under nitrogen flow, and resuspended in ethanol.

the chromatogram at $290 \mathrm{~nm}$ (red trace) at the retention time expected for reduced plastoquinone ( $8.1 \mathrm{~min}$, see below and [14]). This confirms that during the fractionation through the aluminium oxide column, reduced $\mathrm{PQH}_{2}$ gets oxidized [19]. After addition of $\mathrm{NaBH}_{4}$ to reduce $\mathrm{PQ}$ $[10,14]$, the elution peak at 19.2 minutes in the $255 \mathrm{~nm}$ chromatogram (lower panel of Figure 1, black trace) decreased, and the reduced PQ eluted earlier with a retention time of $8.1 \mathrm{~min}$, better detected at 290 $\mathrm{nm}$ (lower, left panel of Figure 1, red trace). Although reduction of the oxidized PQ form upon $\mathrm{NaBH}_{4}$ addition was incomplete, the elution profiles showed peaks with the same retention times and the same qualitative response to reducing conditions as those reported in [14]. 
Citation: Malferrari M, Francia F (2014) Isolation of Plastoquinone from Spinach by HPLC. J Chromatogr Sep Tech 5: 242. doi:10.4172/21577064.1000242

UV absorption spectrum of purified PQ, obtained after collection of the 19.2 minutes retention time HPLC peak, is presented in Figure 2, upper panel. As better evidenced in the oxidized-reduced difference spectrum (lower panel), the absorption profile has a maximum at 255 $\mathrm{nm}$ which is typical of PQ. The oxidized-reduced difference spectrum exhibits isosbestic points at 235, 275, and $310 \mathrm{~nm}$, in good agreement with values reported in the literature [18]. Variations of these values in a limited range (1-2 $\mathrm{nm}$ ) are usually ascribed to small changes in the background absorption of the sample, following the addition of $\mathrm{NaBH}_{4}$ before acquiring the spectrum of the reduced form [18].

The concentration of the HPLC purified PQ was estimated from the oxidized-minus-reduced difference spectrum at $255 \mathrm{~nm}$ (ox-red $\Delta \mathrm{A}_{255 \mathrm{~nm}}$ ), assuming a differential extinction coefficient $\Delta \varepsilon_{255 \mathrm{~nm}}=15 \mathrm{mM}$ ${ }^{1} \mathrm{~cm}^{-1}[14]$ for the difference band. Injection of a known amount of PQ in the analytical HPLC system allowed us to estimate the extraction yield. The yield was found to be $0.023 \mathrm{moles} P Q / \mathrm{mg}$ Chl, slightly less than what reported for established method [8]. In our working conditions with analytical HPLC, we obtained about $30 \mu \mathrm{g}$ of plastoquinone for each $20 \mu \mathrm{L}$ of PQ-containing-fraction injected in the column.

\section{Conclusions}

The involvement of $\mathrm{PQ}$ in photosynthetic electron transport, redox signaling pathways and the recent evidence that $\mathrm{PQ}$ can act as singlet oxygen scavenger under high light stress are renewing the interest of researchers for this cofactor.

In the present work we have shown that PQ isolation can be easily done using partition of chloroplast suspension with methanol:petroleum ether followed by orthogonal chromatographic steps. Since PQ is not commercially available, our procedure is expected to facilitate the production and usage of this molecule for bioassay or quantification purposes in a normally equipped laboratory.

\section{Acknowledgment}

We thank Fabiana Antonioni and Carlotta Pia Cristalli for help in analytical HPLC plastoquinone isolation, and Giovanni Venturoli for critical reading of the manuscript.

F.F. and M.M. acknowledge financial support by University of Bologna, Italy (Grant FARB2012-FFBO122037).

\section{References}

1. Crofts AR, Wraight CA (1983) The electrochemical domain of photosynthesis Biochim Biophys Acta 726: 149-185.

2. Cramer WA, Knaff DB (1990) Energy transduction in biological membranes. A textbook of bioenergetics. Springer.

3. Allen JF, Bennett J, Steinback KE, Arntzen CJ (1981) Chloroplast protein phosphorylation couples plastoquinone redox state to distribution of excitationenergy between photosystems. Nature 291: 25-29.

4. Zito F, Finazzi G, Delosme R, Nitschke W, Picot D, et al. (1999) The Qo site of cytochrome $b_{6} f$ complexes controls the activation of the LHCII kinase. EMBO J 18: 2961-2969.

Citation: Malferrari M, Francia F (2014) Isolation of Plastoquinone from Spinach by HPLC. J Chromatogr Sep Tech 5: 242. doi:10.4172/2157-7064.1000242
5. Pesaresi P Hertle A, Pribil M, Kleine T, Wagner R, et al. (2009) Arabidopsis STN7 kinase provides a link between short- and long-term photosynthetic acclimation. Plant Cell 21: 2402-2423.

6. Pfalz J, Liebers M, Hirth M, Grübler B, Holtzegel U, et al. (2012) Environmental control of plant nuclear gene expression by chloroplast redox signals. Front Plant Sci 3: 1-9.

7. Nowicka B, Kruk J (2012) Plastoquinol is more active than Ît-tocophero in singlet oxygen scavenging during high light stress of Chlamydomonas reinhardtii. Biochim Biophys Acta 1817: 389-394.

8. Barr R, Crane FL (1971) Quinones in Algae and Higher Plants. Meth Enzimo 23: $372-408$

9. Bucke C, Leech RM, Hallaway M, Morton RA (1966) The taxonomic distribution of plastoquinone and tocopherolquinone and their intracellular distribution in leaves of Vicia faba L. Biochim Biophys Acta 112: 19-34.

10. Kruk J (1988) Charge-transfer complexes of plastoquinone and alphatocopherol quinone in vitro. Biophys Chem 30: 143-149.

11. Okayama S (1984) Reversed-phase high-performance liquid chromatography of prenylquinones in green leaves using an electrochemical detector. Plant Cell Physiol 25: 1445-1449.

12. Arnon DI (1949) Copper Enzymes in Isolated Chloroplasts. Polyphenoloxidase In Beta Vulgaris. Plant Physiol 24: 1-15.

13. Venturoli G, Fernandez-Velasco JG, Crofts AR, Melandri BA (1986) Demonstration of a collisional interaction of ubiquinol with the ubiquinolcythocrome c2 oxidoreductase complex in chromatophores from Rhodobacter sphaeroides. Biochim Biophys Acta 851: 340-352.

14. Yoshida K, Shibata M, Terashima I, Noguchi K (2010) Simultaneous determination of in vivo plastoquinone and ubiquinone redox states by HPLCbased analysis. Plant Cell Physiol 51: 836-841.

15. Martinis J, Kessler F, Glauser G (2011) A novel method for prenylquinone profiling in plant tissues by ultra-high pressure liquid chromatography-mass spectrometry. Plant Methods 7: 23.

16. Kruk J, Karpinski S (2006) An HPLC-based method of estimation of the tota redox state of plastoquinone in chloroplasts, the size of the photochemically active plastoquinone-pool and its redox state in thylakoids of Arabidopsis. Biochim Biophys Acta 1757: 1669-1675

17. Kruk J, Strzalka K, Leblanc RM (1992) Monolayer study of plastoquinones,? tocopherolquinone, their hydroquinone forms and their interaction with monogalactosyldiacylglycerol. Charge-transfer complexes in a mixed monolayer Biochim Biophys Acta 1112: 19-26.

18. Henninger MD, Barr R, Crane FL (1966) Plastoquinone B. Plant Physiol 41 696-700.

19. Barr R, Henninger MD, Crane FL (1967) Comparative Studies on Plastoquinone II. Analysis for Plastoquinones A, B, C, and D. Plant Physiol 42: 1246-1254.

Submit your next manuscript and get advantages of OMICS Group submissions

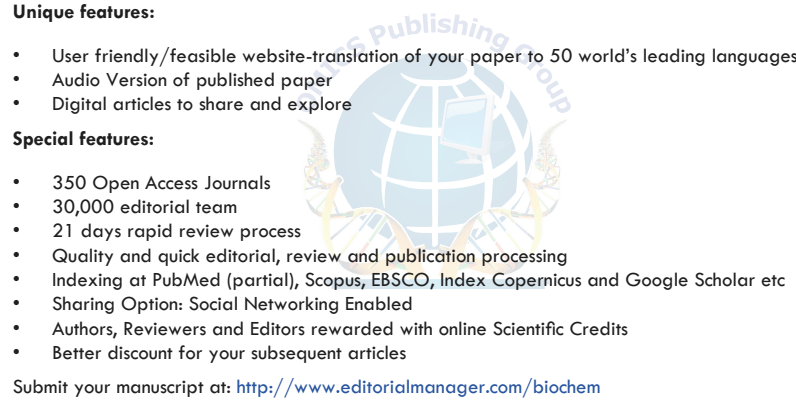

